Cahiers de philosophie de l'université de

\title{
Martin Heidegger : reflets croisés
}

Entretien

\section{Martin Heidegger}

Traducteur : Sylvaine Gourdain

\section{OpenEdition}

\section{Journals}

Édition électronique

URL : https://journals.openedition.org/cpuc/290

DOI : $10.4000 /$ cpuc. 290

ISSN : 2677-6529

\section{Éditeur}

Presses universitaires de Caen

\section{Édition imprimée}

Date de publication : 1 novembre 2018

Pagination : 17-20

ISBN : 978-2-84133-904-4

ISSN : $1282-6545$

Référence électronique

Martin Heidegger, "Martin Heidegger : reflets croisés », Cahiers de philosophie de l'université de Caen [En ligne], 55 | 2018, mis en ligne le 01 novembre 2019, consulté le 12 février 2023. URL : http:// journals.openedition.org/cpuc/290; DOI : https://doi.org/10.4000/cpuc.290

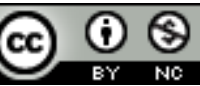

Creative Commons - Attribution - Pas d'Utilisation Commerciale 4.0 International - CC BY-NC 4.0 https://creativecommons.org/licenses/by-nc/4.0/ 


\section{Martin Heidegger : reflets croisés ${ }^{1}$}

Hisamatsu - Je tiens à vous remercier très sincèrement d'avoir pris la direction du séminaire hier soir, malgré toutes vos obligations. Soyez remercié également pour votre hospitalité.

Heidegger - Des personnes de plusieurs disciplines différentes étaient présentes. Ce n'était donc pas si facile.

Hisamatsu - J'ai été très heureux que cela mène à toutes sortes d'échanges.

Heidegger - Il faudrait consacrer plusieurs jours à un tel séminaire.

Hisamatsu - C'est vrai. Ce thème recouvre vraiment des questions difficiles. De quels domaines venaient les personnes présentes hier?

Heidegger - M. Bröse est le président de la Société des beaux-arts. En réalité, il est juriste. Il a assisté à l'un de mes cours il y a 33 ans.

Hisamatsu - Est-ce qu'il peint aussi lui-même?

Heidegger - Non, il ne peint pas. Il travaille à la Société des beaux-arts, il s'intéresse à la théorie de la peinture et soutient de jeunes artistes. À part lui, il y avait aussi Jürg Spiller, le célèbre peintre abstrait.

Hisamatsu - N'était-il pas élève de Paul Klee?

Heidegger - Klee était professeur de peinture au Bauhaus.

Hisamatsu - Klee est connu même au Japon.

Heidegger - Spiller connaissait Paul Klee.

1. Cet entretien s'est tenu dans la maison de Heidegger le lendemain de l'entretien précédent. Parce qu'il a été traduit en allemand à partir d'une transcription japonaise de l'enregistrement, il figure comme $\$ 290$ dans les ajouts du tome 16 de l'édition intégrale, qui regroupent des textes qui ne sont pas authentiquement de la main de Heidegger. Nous remercions Arnulf Heidegger et les éditions Klostermann pour nous avoir donné l'autorisation de publier ces pages. ( $)$ M. Heidegger, "Wechselseitige Spiegelung», in Gesamtausgabe, Francfort-sur-le-Main, V. Klostermann, t. 16, Reden und andere Zeugnisse eines Lebensweges, 2000, p. 776-78o. 
Hisamatsu - J'ai moi aussi eu l'occasion de voir des tableaux de Klee.

Heidegger - Vous en avez vu? J'ai plus d'estime pour Paul Klee que pour Picasso. Selon moi, Paul Klee est un peintre plus important que Picasso.

Hisamatsu - Alcopley avait-il des contacts avec Klee?

Heidegger - Je ne sais pas s'ils ont eu des contacts. Il est vrai qu'ils se ressemblent sur bien des points, mais Paul Klee est mort dès 1940. - L'éditeur de ce livre [Paul Klee. Leben und Werk] est Spiller. Il viendra probablement ce soir aussi. Cette peinture (une peinture du temple Jisho-ji), c'est le directeur de la compagnie maritime Bremer Lloyd qui me l'a rapportée du Japon.

Hisamatsu - Ceci, c'est le temple Toguji, on l'appelle Togudo. Il abrite le premier salon de thé japonais. On peut dire que la Voie du thé a commencé au temple de Ginkakuji. Le shogun Yoshimasa a chargé Murata Shuko de développer la Voie du thé.

Heidegger - Voici un recueil d'œuvres de Klee qui est paru récemment ${ }^{2}$. Hisamatsu - C'est vraiment un livre magnifique. Ce tableau a quelque chose d'oriental. Mon tableau préféré est L'escargot [Die Schnecke, 1924]. Quel tableau de Klee vous plaît?

Heidegger - Hum, j'aime le dessin Lune d'argent [Silbermondgeläute, 1931]. Êtes-vous déjà allé à Berne? Le fils de Klee y vit. Il possède de nombreuses œuvres de Klee.

Tsujimura - Malheureusement, nous n'avons fait que passer à côté en train.

Heidegger - Dommage! Mais vous avez déjà vu beaucoup de choses si vous avez pu contempler la Jungfrau et quelques autres lieux avant de venir ici.

Hisamatsu - Voilà un bon tableau. Sa couleur correspond au principe du sabi. Et il n'y a rien d'artificiel dans son tracé.

Heidegger - Moi aussi, j'aime ce tableau. L'original se trouve à Berne. Il est intitulé Sainte à une fenêtre [Heilige aus einem Fenster, 1940-1956]. Klee l'a peint lui aussi dans les dernières années de sa vie.

Hisamatsu - D'une certaine façon, ce tableau a quelque chose d'une calligraphie japonaise.

Heidegger - Oui, c'est vrai. - Klee était aussi un musicien important. Il aurait dit que les véritables maîtres sont Mozart et Beethoven.

Hisamatsu - J'aimerais beaucoup vous entendre parler de vos idées les plus récentes.

2. P. Klee, Im Zwischenreich. Aquarelle und Zeichnungen von Paul Klee, W. Haftmann (éd.), Cologne, M. DuMont Schauberg, 1957. 
Heidegger - J'ai de nouvelles idées, mais elles ne sont pas achevées. Je les ai mises par écrit, certes, mais je ne les ai pas encore présentées devant un auditoire. Je n'avance vraiment que très lentement, pas à pas. Cela tient également au problème du langage. Cette nouvelle pensée transparaît aussi en partie dans la conférence que j'ai prononcée récemment ${ }^{3}$; elle constitue l'arrière-plan de la conférence, mais elle n'y est pas encore exprimée de façon tout à fait claire. J'aimerais surmonter les préjugés portant sur le langage. La manière occidentale de comprendre le langage à partir de la grammaire est soumise à la domination de l'ontologie aristotélicienne, mais aussi de l'ontologie grecque. Mais le langage d'un poète ne peut être compris à l'aide de cette conception grammaticale du langage. J'en ai déjà parlé. L'ancien japonais, qui n'avait encore eu aucun contact avec les langues indo-européennes, soulève certainement de ce point de vue quelques questions intéressantes. Un jour, Klee a écrit ceci - cela ressemble à un poème: "Les oiseaux sont enviables, / ils évitent / de penser au tronc et aux racines, / et, satisfaits, ils se balancent toute la journée, agiles / et chantent sur les dernières des ramilles».

Hisamatsu - Ce dont vous parlez ici, on le trouve aussi dans des entretiens zen. Dans le zen, le langage est considéré comme une expression entièrement libre. Si libre que c'est seulement après qu'il a été prononcé qu'une grammaire se constitue.

Heidegger - Mes réflexions vont elles aussi dans cette direction.

Hisamatsu - Je souhaite vraiment que, dans tous les cas, vous développiez ces idées.

Heidegger - Mon élève Buchner est parti au Japon. Je vous prie de bien vouloir le soutenir et l'encadrer.

Hisamatsu - J'aimerais moi aussi apprendre de M. Buchner. J'espère que son séjour contribuera au développement d'une pensée orientaleoccidentale. Je souhaite qu'un jour l'Orient inclue en lui l'Occident et qu'à l'inverse l'Occident inclue en lui l'Orient, et que cela conduise ainsi au déploiement d'une pensée orientale-occidentale.

Heidegger - Je vous remercie pour ces paroles. Je trouve qu'une telle rencontre entre l'Orient et l'Occident est plus importante que des contacts économiques et politiques.

Hisamatsu - Je suis du même avis. Probablement de telles rencontres engendreront-elles des transformations qui toucheront aussi la politique.

3. Il s'agit de la conférence intitulée «Le mot» (Acheminement vers la parole), prononcée le 11 mai 1958 à Vienne en présence de Hisamatsu. 
Heidegger - Naturellement. L'inverse n'est pas possible. On ne peut avancer en partant de la politique et de l'économie. Il faut commencer depuis un lieu plus profond.

Hisamatsu - En effet, je suis tout à fait d'accord. Vos paroles me sont un grand présent pour mon retour au Japon.

Heidegger - Pour autant que je sache, le séminaire d'hier a fait grande impression sur les personnes présentes. Et c'est le cas aussi de notre entretien d'aujourd'hui.

Hisamatsu - Mais je vous en prie... merci! J'aimerais beaucoup rapporter chez moi quelques lignes écrites de votre main. Un poème que vous aimez, quelque chose que vous avez écrit vous-même ou quelque chose d'autre.

Heidegger - [Va chercher une édition limitée et hors commerce de Johann Peter Hebel - L'ami de la maison.] Je vais écrire en lettres allemandes, et non latines.

Hisamatsu - Ah, merci beaucoup!

Tsujimura - Traduit en japonais, cela veut dire: «En souvenir amical de votre visite à Freiburg». [Heidegger dédicace également son petit livre Le chemin de campagne, et l'offre à Hisamatsu.]

Hisamatsu - Grand merci pour tous ces présents! [Montrant une photo de Heidegger dans l'édition limitée de Johann Peter Hebel.] De quelle année date cette photo?

Heidegger - De 1955. 